\title{
VALIDATION OF HF RADAR MEASUREMENTS
}

\author{
By Rick D. Chapman and Hans C. Graber
}

\section{But oceanographers}

. . may reasonably

ask how accurate

can such remote

measurements be

made?
$\mathrm{H}_{\mathrm{F}}$ measuring surface currents. They provide an unparalleled window into the spatial variations of near-surface currents. But oceanographers who are more accustomed to measuring currents with instruments that actually get wet may reasonably ask how accurate can such remote measurements be made? And while this is an easy and obvious question to ask, it is an interestingly difficult question to answer.

We have been studying the accuracy of the OSCR HF radar system through analysis of data from the Office of Naval Research (ONR)-sponsored High-Resolution Remote-Sensing Experiment that was conducted off Cape Hatteras. North Carolina during the summer of 1993. This experiment provided a unique opportunity to examine the complex questions of HF radar accuracy. Along with several weeks of HF radar data, we had access to multiple in situ measurements of current from both moored and ship-based devices. In a series of analyses. we have attempted to validate the HF current measurements through comparison with the in situ data. The key has been to examine the temporal and spatial variations within these data in order to distinguish the sources of the underlying differences between the systems we compare.

\section{Comparisons with In Situ Instruments}

When evaluating the accuracy of a new instrument. the typical procedure is to compare side-byside measurements made with both the new instrument and an older instrument of known accuracy. It is important in such a comparison that the two instruments are measuring the same physical quantities, but this is a problem in evaluating the accuracy of an $\mathrm{HF}$ radar. The canonical HF radar measures near surface currents integrated over the upper $50 \mathrm{~cm}$, averaged over a $1-\mathrm{km}$ square and averaged over a 10 -min period. Typical in situ current meters measure currents at fixed depths that are typically greater than the $\mathrm{HF}$ radar's effective depth, at essentially a single point in space and

Rick D. Chapman, Applied Physics Laboratory. Johns Hopkins University, Johns Hopkins Road, Laurel, MD 20723, USA. Hans C. Graber, Rosenstiel School of Marine and Atmospheric Science, University of Miami. Miami. FL 33149. USA. offer fast response. The differences observed when these systems are compared are a result of differences in the measured quantity combined with the sampling techniques and inaccuracies of the instruments themselves. This makes it difficult to isolate the accuracy of the HF radar from other sources of observed difference.

The first pioneers in this field compared HF radar measurements with drifters (Stewart and Joy, 1974; Barrick et al., 1977; Frisch and Weber, 1980). These comparisons were limited by the paucity of data and limits on the spatial and temporal coverage of the drifters, but they served to provide an upper bound on the errors of the HF system of $15-27 \mathrm{~cm} / \mathrm{s}$. Some later investigations compared the HF radar data with bottom-mounted Acoustic Doppler Current Profilers (ADCPs) or moored instruments (Holbrook and Frisch, 1981; Leise, 1984; Porter et al., 1986; Matthews et al.. 1988; Shay et al., 1995), finding differences ranging from 9 to $17 \mathrm{~cm} / \mathrm{s}$. Prandle (1991) performed a similar study but limited the comparisons to tidal and lower frequencies. The argument was made that these low temporal frequencies imply low spatial frequencies, making the in situ measurements made at a point more comparable to the area- and time-averaged HF radar measurements.

In our initial study (Chapman et al., 1997) we compared in situ measurements from shipmounted and towed ADCPs with HF radar measurements. We began by averaging the in situ data into 20-min samples, corresponding to the OSCR sampling period. A pseudo time series was then constructed from the time series of OSCR current maps, by tracking the movement of the ship through the OSCR measurement domain. Thus we constructed a subset of the OSCR data that was directly comparable with the in situ data set.

The direct comparisons of HF and in situ current measurements made in this way, an example of which is shown in Figure 1, indicate differences of $8-15 \mathrm{~cm} / \mathrm{s}$. But from this limited form of comparison, it is impossible to determine how these differences are apportioned between errors in the HF radar, errors in the in situ sensors, or differences in the measured quantities.

We have improved on these analyses by creating a model of the errors in the HF radar, and ex- 
amining how these errors differ from those of the in situ sensors. This simple model allows us to separate out the various sources of difference. We began by considering the geometric dependence of errors in the HF radar.

\section{Geometric Model of HF Radar Errors}

As described elsewhere in this issue, the HF radar estimates vector currents by measuring the radial currents from two separate stations. These two radial estimates are then combined to form estimates of the vector current at each point in the measurement domain. It is reasonable to assume that each of the stations measures the radial velocity to the same levels of accuracy. We will further assume that, with proper installation of the HF radar systems (in particular the proper physical and electrical alignment of the phased array antennas), these radial velocity errors are relatively position independent, at least for those ranges where the signal-to-noise ratio is sufficiently high. It then turns out that combining these two radial velocity measurements into a vector current measurement imposes a position-dependent error on the vector components.

This is most easily seen by considering Figure 2 , which indicates the station locations and coverage area of the OSCR system for the High-Res experiment. Assume that each radial velocity measurement has an associated root-mean-square (rms) error of $\sigma_{r}$. Consider the errors in the North and East current components determined at a point at the far extreme of the map, due East of the stations. As the range increases, the East component of the velocity takes the form of the average of the two radial components, and thus the rms error in the east component approaches $\sigma_{r} / \sqrt{2}$. In contrast, the North component of the current is related to the difference of the radial components, a difference of large numbers, and so we would expect the errors to be significantly larger than $\sigma_{r}$.

We in fact have worked out a model for the positional dependency of the rms errors in the current components, the results of which are shown by the contours in Figure 2. We write that the errors in a current component are given by

$$
\sigma_{n}=G D O P_{n} \bullet \sigma_{r}, \sigma_{e}=G D O P_{e} \bullet \sigma_{r}
$$

where $\sigma_{n}$ and $\sigma_{e}$ are the rms errors in the north or east directions, $\sigma_{r}$ is the radial velocity error from a single station, and $G D O P_{n}$ and $G D O P_{e}$ are the Geometric Dilution of Precisions, factors determined by our model. (The GDOP terminology was borrowed from the Global Positioning System (GPS) community, e.g., see Wells et al., 1986).

The contours of constant GDOP in Figure 2 indicate that the errors in the north component of the HF radar current measurements will be larger than in the eastern component. Furthermore, the errors in the HF radar determination of the north component of the current vary sig-

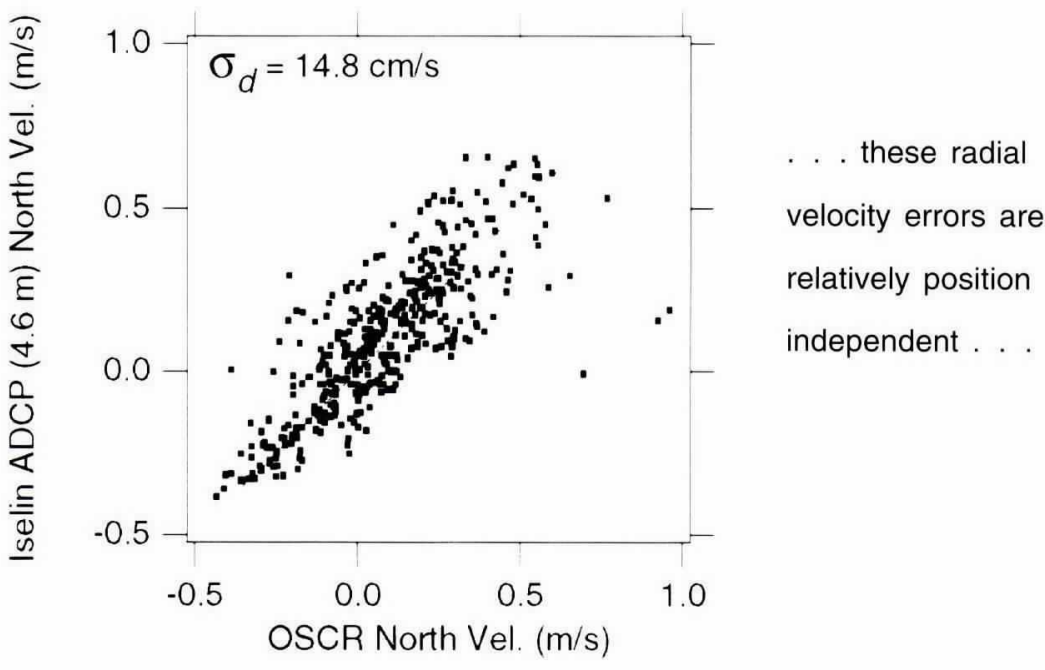

Fig. 1: Comparison of north component of nearsurface current as determined by a ship-mounted $A D C P$ at $4.6 \mathrm{~m}$ depth and OSCR. The rms difference between the estimates is $14.8 \mathrm{~cm} / \mathrm{s}$. The dotted line is a line of equal velocity.

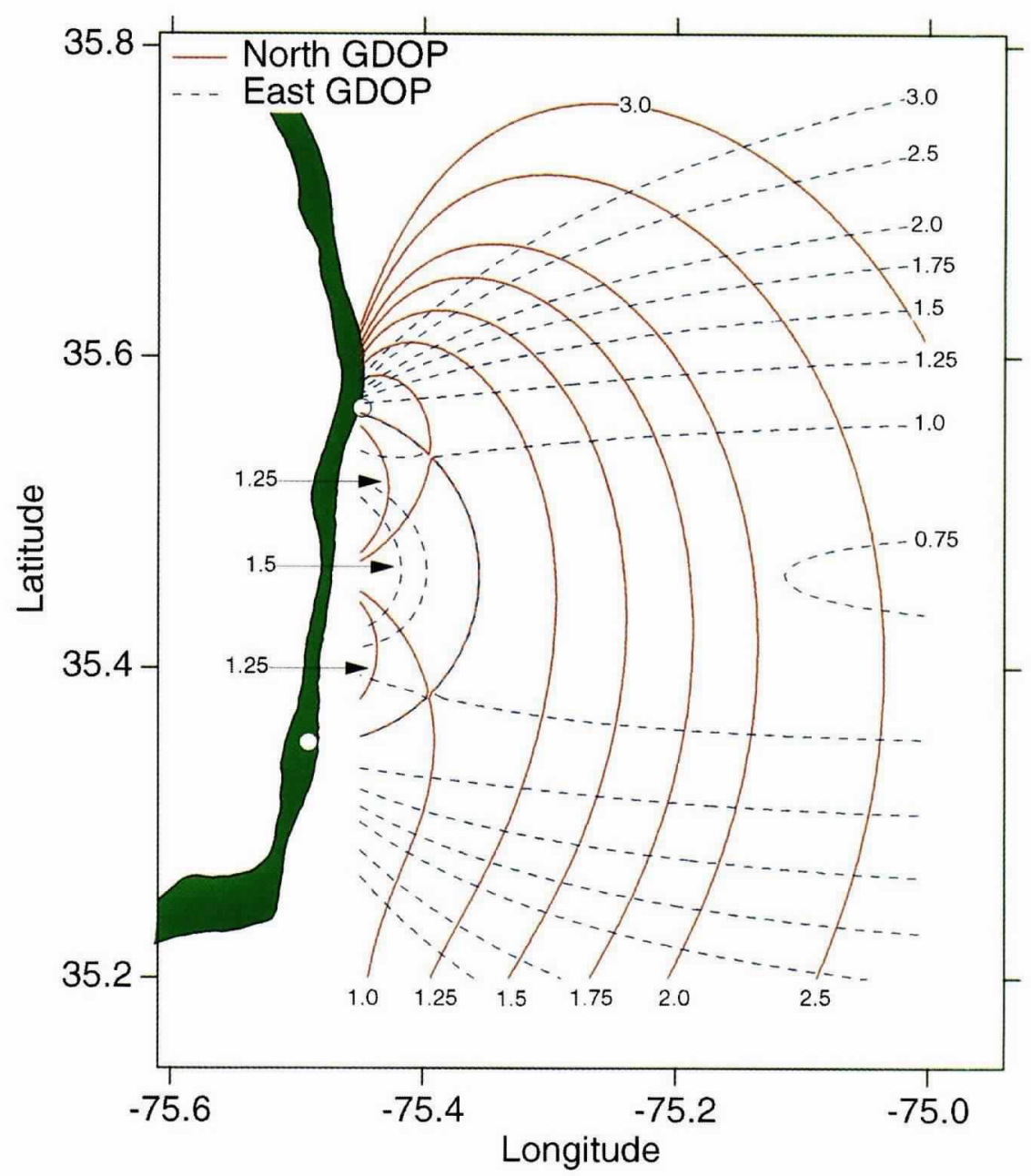

Fig. 2: Map of the North (solid red lines) and East (dashed blue lines) Geometric Dilution of Precision (GDOP) for the OSCR measurement domain. The circles along the coast designate the OSCR sites, and the gray dots indicate the OSCR measurement locations. 


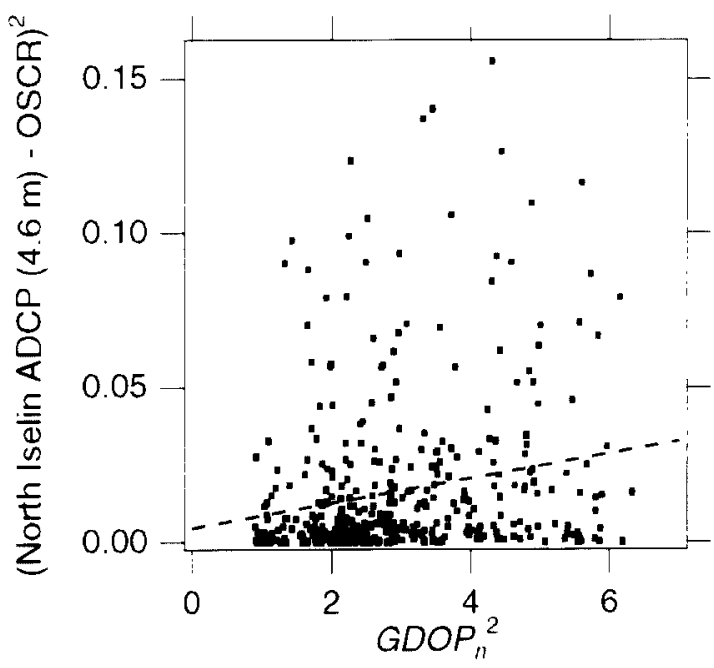

Fig. 3: Variations of the square of observed current component differences with GDOP'. The dashed line represents a least-squares linear fit to these data, the slope of which indicates $\sigma_{\mathrm{r}}$, the noise in the radial component of OSCR currents.

\section{The alignment of}

the mean differences

with the Gulf Stream

is evident . . .

\section{Error Budget}

As a further check, the error budget above can be further expanded, with the individual terms $\sigma_{\text {physccs }}^{2}$ each accounted for separately. This can be done either from the data or from geophysical models.

The data-centric approach examines the structure functions of the current, or the expected rms value associated from currents measured at two different locations, depths or times, as a function of distance, depth, or lag. Figure 4 contains the spatial structure function of the expected differences as estimated from the OSCR data sets and several moored current meters.

While Figure 4 provides an estimate of the magnitude of the differences attributable to spatial inhomogenieties in the currents, these differences do in fact vary in a complex manner. This is shown in Figure 5, which plots contours of the rms differences (black), along with the magnitude of the complex correlation coefficient (red), for the OSCR currents referenced to a single OSCR cell near the middle of the measurement domain. The alignment of the mean differences with the Gulf Stream is evident, along with associated cross-stream decorrelation of the current fluctuations.

Alternatively, geophysical models can provide estimates of the expected differences, by modeling such physical processes as horizontal current variability, the Stokes drift, Ekman drift, and currentinduced baroclinicity.

Graber et al. (1997) combined these apwhere $\sigma_{\text {diff }}$ is the rms difference between the measurements, $\sigma_{H F}$ is the rms error in the HF radar measurement, $\sigma_{\text {in situ }}$ is the rms error in the in situ measurement, and $\sigma_{\text {physics }}$ is the rms difference in the physical parameters measured by the $\mathrm{HF}$ radar and in situ instruments. We have assumed here that the errors in the in situ measurements and the rms differences in the physical parameters are uncorrelated with the rms errors in the HF radar measurements, an assumption that we have verified by statistical analysis of our data sets.

Our problem is thus reduced to finding $\sigma_{H F}^{2}$ given the observed $\sigma_{\text {diff. }}^{2}$. Our model suggests that the observed errors should be expressible as

$$
\sigma_{\text {diff }}^{2}=\sigma_{r}^{2} \cdot G D O P^{2}+\sigma_{o t h e r}^{2}
$$

Figure 3 is a plot of the squares of the observed differences in the north current component versus the square of $G D O P_{n}$ as determined by the model and the in situ measurement location. Although these data are obviously noisy, a least-squares linear fit does suggest that $\sigma_{r}$ is of the order of 7-8 $\mathrm{cm} / \mathrm{s}$, a value comparable with the $\mathrm{rms}$ noise in the in situ sensors. Although all of this might seem a bit round about, we know of no other way of separating the accuracy of the HF radar from the other sources of differences. proaches to examine how much of the total observed variance can be accounted for. They con-

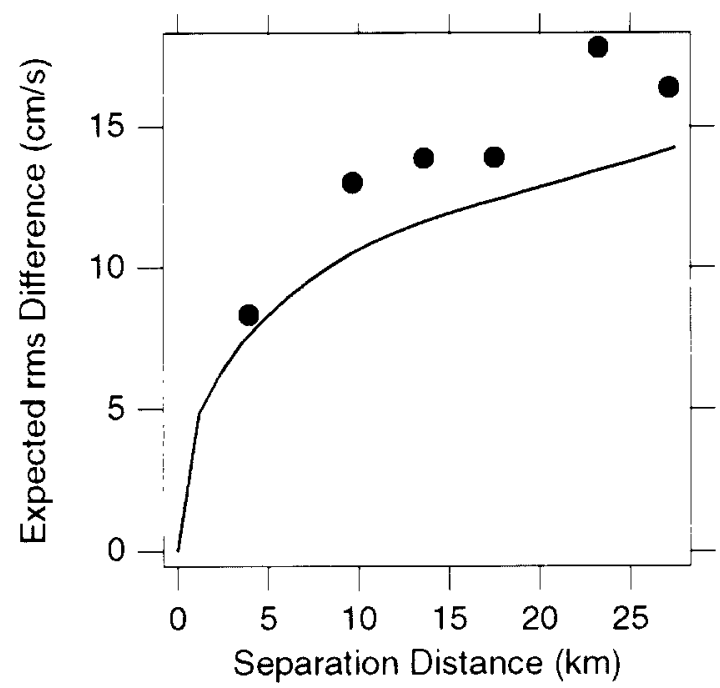

Fig. 4: Expected rms differences between nearseparation. The solid line show's estimates of the rms differences for OSCR versus cross-shelf lag, and the solid dots show values for pairs of moored current meters. surface observations as a function of cross-shelf 
cluded that $40-60 \%$ of the observed rms differences between the radar-derived surface current and the near-surface current measurements can be explained. Their study indicated that differences due to spatial separation and baroclinicity appear to be comparable with the errors in the radar measurements themselves. However, in strongly wind-forced ocean conditions, the Stokes and Ekman drift terms can easily dominate these differences.

\section{Conclusion}

Direct comparisons of HF radars with in situ instruments place an upper bound on the accuracy of the radar-derived current measurements of $10-15 \mathrm{~cm} / \mathrm{s}$. These estimates can be improved by examining the spatial dependence of the variability of observed current differences. This procedure suggests that the radar-derived radial velocity errors are more likely on the order of 7-8 cm/s. Further analysis of the underlying causes of differences suggests that most of the differences can be accounted for in terms of surface current variability in space, depth, and time, as well as errors in the in situ and radar-derived currents. We conclude that when properly deployed, HF radars can accurately measure ocean surface currents, providing a unique tool for near-shore monitoring.

\section{Acknowledgments}

The authors gratefully acknowledge the contributions of their other collaborators in this research: L.K. Shay, J.B. Edson, A. Karachintsev, C.L. Trump, D.B. Ross, and B.K. Haus. This research was supported, in part, by grants N0003991-C-0001 (R.D. Chapman) and N00014-91-J1775 (H.C. Graber) from the Office of Naval Research.

\section{References}

Barrick, D.E., M.W. Evans and B.L. Weber, 1977: Ocean surface currents mapped by radar. Science, 198, 138-144.

Chapman, R.D., L.K. Shay, H.C. Graber, J.B. Edson, A. Karachintsev, C.L. Trump and D.B. Ross, 1997: On the accuracy of HF radar surface current measurements: intercomparisons with ship-based sensors. J. Geophys. Res., 102, 18,737-18,748.

Frisch, A.S. and B.L. Weber, 1980: A new technique for measuring tidal currents by using a two-site HF doppler radar system. J. Geophys. Res., 85, 485-493.

Graber, H.C., B.K. Haus, L.K. Shay and R.D. Chapman, 1997: HF radar comparisons with moored estimates of current speed and direction: expected differences and implications. J. Geophys. Res., 102, 18,749-18,766.

Holbrook, J.R. and A.S. Frisch, 1981: A comparison of nearsurface CODAR and VACM measurements in the Strait of Juan De Fuca, August 1978. J. Geophys. Res., 86, 10908-10912.

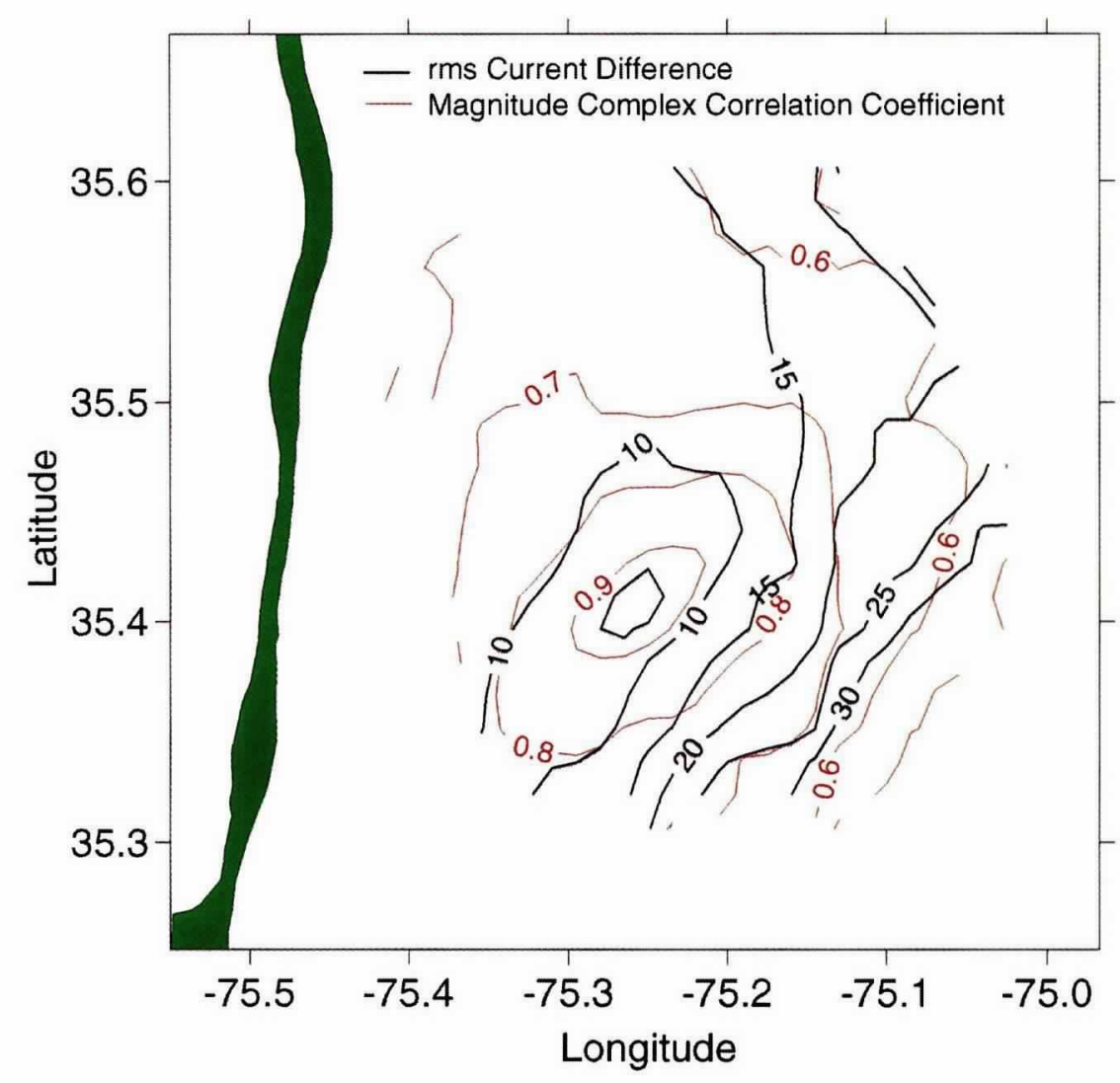

Fig. 5: Spatial variability of OSCR surface currents calculated with respect to a single OSCR cell. The black contours are the rms difference for speed, and the red contours are the magnitude of the complex correlation coefficient at zero temporal lag.

Leise, J.A., 1984: The analysis and digital signal processing of NOAA's surface current mapping system. IEEE $J$. Oceanic Eng., OE-9, 106-113.

Matthews, J.P, J.H. Simpson and J. Brown, 1988: Remote sensing of shelf sea currents using a high-frequency ocean surface current radar system. J. Geophys. Res., 93, 2303-2310.

Porter, D.L., R.G. Williams and C.R. Swassing II, 1986: CODAR intercomparison: Delaware Bay 1984. Proceedings of IEEE Third Working Conference on Current Measurement, 36-44, Pergamon Press, UK.

Prandle, D., 1991: A new view of near-shore dynamics based on observations from HF radar. Prog. Oceanogr., 27, 403-438.

Shay, L.K., H.C. Graber, D.B. Ross and R.D. Chapman, 1995: Mesoscale ocean surface current structure detected by HF radar. J. Atmos. Ocean. Tech., 12, 881-900.

Stewart, R.H. and J.W. Joy, 1974: HF radio measurements of surface currents. Deep Sea Res., 21, 1039-1049.

Wells, D.E., N. Beck, D. Delikaraoglou, A. Kleusberg, E.J. Krakiwsky, G. Lachapelle, R.B. Langley, M. Nakiboglu, K.P. Schwarz, J.M. Tranquilla and P. Vanicek, 1986: Guide to GPS Positioning. Canadian GPS Associates, Fredericton, N.B., Canada. $\square$
Direct comparisons

of $\mathrm{HF}$ radars with in situ instruments place an upper bound on the accuracy of the radar-derived current measurements . . . 